PROCEEDINGS OF THE

AMERICAN MATHEMATICAL SOCIETY

Volume 131, Number 7, Pages 2103-2108

S 0002-9939(03)07032-1

Article electronically published on February 5, 2003

\title{
THE IDEAL PROPERTY IN CROSSED PRODUCTS
}

\author{
CORNEL PASNICU
}

(Communicated by David R. Larson)

\begin{abstract}
We describe the lattice of the ideals generated by projections and prove a characterization of the ideal property for "large" classes of crossed products of commutative $C^{*}$-algebras by discrete, amenable groups; some applications are also given. We prove that the crossed product of a $C^{*}$-algebra with the ideal property by a group with the ideal property may fail to have the ideal property; this answers a question of Shuzhou Wang.
\end{abstract}

\section{INTRODUCTION}

A $C^{*}$-algebra is said to have the ideal property if its ideals are generated (as ideals) by their projections (in this paper, by an ideal we shall mean a closed, two-sided ideal).

The class of the $C^{*}$-algebras with the ideal property is important because it includes the $C^{*}$-algebras of real rank zero $(\underline{\mathrm{BPe}})$ and also the simple, unital $C^{*}$ algebras. The ideal property has been studied in $[\mathrm{Pa} 1-[\mathrm{Pa} 9,[\mathrm{PaR}],[\mathrm{S}]$. On the other hand, the crossed products $\left([\mathrm{Pe})\right.$ represent a valuable source of interesting $C^{*}$ algebras, related to the domain of the dynamical systems. Many important nuclear, simple, unital, separable $C^{*}$-algebras generated by a subset of elements or many $A H$ algebras are in fact crossed products of commutative, separable $C^{*}$-algebras by discrete, countable, amenable groups (e.g. the irrational rotation algebras, the Bunce-Deddens algebras and others (see [B1])). It became clear that the nuclear crossed products with the ideal property will play a central role in Elliott's project of the classification of the nuclear, separable $C^{*}$-algebras by invariants including $K$-theory ([EII]). Therefore, it is clearly important to know when a crossed product has the ideal property. Our main interest is in the case when the group is discrete and it acts on a commutative $C^{*}$-algebra. For a discrete, countable, amenable group $G$ and a commutative, separable $C^{*}$-algebra $A$ let $\alpha$ be an action of $G$ on $A$ which induces a free action of $G$ on the spectrum of $A$. Based mainly on results in $[\mathrm{EH}$, [GL] and [Z], we identify the lattice of the ideals generated by projections of $A \rtimes G$ with a lattice of $\alpha$-invariant ideals of $A$ (see Theorem 2.1 below), which allows us to characterize the ideal property for $A \underset{\alpha}{\rtimes} G$ (see Corollary 2.2 below). Using this result, we deduce that if in addition the spectrum of the $C^{*}$-algebra $A$ is zero dimensional, or if $A=C(X)$, where $X$ is a compact, Hausdorff topological space and every

Received by the editors February 1, 2002.

2000 Mathematics Subject Classification. Primary 46L05; Secondary 46L55.

Key words and phrases. $C^{*}$-algebra, the ideal property, crossed product.

(C)2003 American Mathematical Society 
closed, $\alpha$-invariant subset of $X$ is also open, it follows that $A \rtimes G$ has the ideal property (see Corollary 2.3 and Corollary 2.4 below). In $[\mathrm{PaR}$ w we proved, jointly with M. Rørdam, that the minimal tensor product of two $C^{*}$-algebras each having the ideal property does not necessarily have the ideal property. On the other hand, if $(A, G, \alpha)$ is a $C^{*}$-dynamical system, then one may visualize the crossed product $A \rtimes G$ as a skew tensor product between $A$ and $C^{*}(G)$, since if $\alpha$ is trivial, then $A \stackrel{\alpha}{\rtimes} G \cong A \otimes_{\max } C^{*}(G)$. We answer a question on Shuzhou Wang, proving that there are crossed products of $C^{*}$-algebras with the ideal property by groups $G$ with the ideal property (i.e., $C^{*}(G)$ has the ideal property; see Definition 2.5 below) that do not have the ideal property (see Theorem 2.6 below).

If $A$ is a $C^{*}$-algebra, the fact that $I$ is an ideal (closed, two-sided) of $A$ will be denoted by $I \triangleleft A$. The projections of a $C^{*}$-algebra $A$ will be denoted by $\mathcal{P}(A)$. Let $G$ be a locally compact group. $G$ is amenable if there is a left invariant mean on $L^{\infty}(G)$ (see $[\mathrm{Pe}]$ ). Note that if $G$ is abelian or if it is compact, then $G$ is amenable ([] $)$. For the definition of $C^{*}(G)$, the group $C^{*}$-algebra of a locally compact group $G$, see [Pe, 7.1.5]. Let $(G, X)$ be a topological transformation group. Recall that $G$ is said to act freely on $X$ (or we say that the action of $G$ on $X$ is a free action) if for every $x \in X$ and for every $g \in G, g$ different from the neutral element of $G$, we have that $g x \neq x$. Recall also that a $C^{*}$-dynamical system is a triple $(A, G, \alpha)$ consisting of a $C^{*}$-algebra $A$, a locally compact group $G$ and a continuous homomorphism $\alpha$ of $G$ into the group $\operatorname{Aut}(A)$ of automorphisms (i.e., *-automorphisms) of $A$ equipped with the topology of pointwise convergence.

Let $(A, G, \alpha)$ be a $C^{*}$-dynamical system, where $G$ is a discrete group with neutral element $e$. Consider an injective $*$-representation $\pi: A \rightarrow \mathcal{B}(H)$. We shall identify $A$ with its image by $\pi$ and $A \underset{\alpha, r}{\rtimes} G$ with the norm closure of $(\tilde{\pi} \times U)\left(l^{1}(G, A)\right)$ in $\mathcal{B}\left(l^{2}(G, H)\right)$, where

$$
\begin{gathered}
(\tilde{\pi}(a) x)(g)=\pi\left(\alpha_{g^{-1}}(a)\right) x(g), \\
\left(U_{h} x\right)(g)=x\left(h^{-1} g\right)
\end{gathered}
$$

for each $a \in A, g, h \in G$ and $x \in l^{2}(G, H)$ (see [Pe, Theorem 7.7.5]). We will denote by $E: A \underset{\alpha, r}{\rtimes} G \rightarrow A$ the canonical conditional expectation given by

$$
E\left(a U_{g}\right)=\left\{\begin{array}{c}
a, \text { if } g=e \\
0, \text { if } g \neq e
\end{array}\right.
$$

where $a \in A, g \in G$. If $M \subseteq A \rtimes G$, we shall denote by $\langle M\rangle$ the (closed and two-sided) ideal of $A \underset{\alpha}{\rtimes} G$ generated by $M$. Suppose now that $A=C(X)$, where $X$ is a compact, Hausdorff topological space. A subset $F$ of $X$ is called $\alpha$-invariant if it is invariant for the action of $G$ on $X$ canonically induced by $\alpha$.

As usual, $\mathbb{T}=\{z \in \mathbb{C}:|z|=1\}$ and $\mathcal{K}$ denotes the $C^{*}$-algebra of compact operators on $l^{2}(\mathbb{N})$.

An $A F$ algebra is an inductive limit $C^{*}$-algebra of the form $\lim _{\rightarrow} A_{n}$, where for each $n \in \mathbb{N}, A_{n}$ is a finite-dimensional $C^{*}$-algebra $([\overline{\mathrm{Br}}])$. 


\section{RESULTS}

Theorem 2.1. Let $(A, G, \alpha)$ be a $C^{*}$-dynamical system such that the $C^{*}$-algebra $A$ is commutative and separable and the group $G$ is discrete, countable and amenable. Suppose that $\alpha$ induces a free action of $G$ on the spectrum of $A$. Then, the map

$$
\theta:\{I \triangleleft A \mid I \text { is } \alpha \text {-invariant }\} \rightarrow\{J \mid J \triangleleft \underset{\alpha}{A \rtimes G}\}
$$

given by

$$
\theta(I):=\underset{\alpha}{\rtimes \rtimes} G
$$

is a lattice isomorphism and it induces canonically a lattice isomorphism

$$
\begin{aligned}
& \left\{I \triangleleft A | I \text { is } \alpha \text { -invariant and } I \subseteq \left\langle\mathcal { P } \left(\underset{\alpha}{\left.\left.\left.I_{\alpha} G\right)\right\rangle\right\}}\right.\right.\right. \\
& \quad \cong\{J \triangleleft A \underset{\alpha}{\rtimes} G \mid J \text { is generated by projections }\} .
\end{aligned}
$$

Proof. For every $x \in \underset{\alpha, r}{A} G$ and every $g \in G$, define $x_{g}:=E\left(x U_{g}^{*}\right)$. For an arbitrary $M \subseteq A$, denote $\mathcal{I}(M)=\left\{x \in A \underset{\alpha, r}{\rtimes} \mid x_{g} \in M\right.$ for every $\left.g \in G\right\}$ (see [Z] 4.15]). Note that since $G$ is amenable, $\underset{\alpha, r}{x_{x} G}=\underset{\alpha}{A \rtimes G}$ ([Z, Théorème 5.1]). Since $A$ is separable and $G$ is a countable, amenable group acting freely on the spectrum of $A$, then it follows by [EH, Corollary 5.16] (as in [Z, Théorème 5.15]) that

$$
\Phi:\{I \triangleleft A \mid I \text { is } \alpha \text {-invariant }\} \rightarrow\{J \mid J \triangleleft A \rtimes \underset{\alpha}{\alpha}\},
$$

defined by

$$
\Phi(I):=\mathcal{I}(I)
$$

for each $\alpha$-invariant ideal $I$ of $A$, is an order isomorphism. Since $G$ is amenable, it follows by $[\mathrm{Z}, 5.10]$ that for each $\alpha$-invariant ideal $I$ of $A$, we have that

$$
\mathcal{I}(I)=\langle I\rangle \text {. }
$$

But, by [GL, Proposition 3.11 (ii)] it follows that $\langle I\rangle=I \rtimes \underset{\alpha}{\rtimes}$ for every $\alpha$-invariant ideal $I$ of $A$. Again using the fact that $G$ is amenable, by a result of E.C. Gootman and A.J. Lazar (GL, Theorem 3.4]) it follows that an ideal $J$ of $A \rtimes G$ is $\hat{\alpha}$-invariant if and only if $J=\underset{\alpha}{\rtimes} G$, for some unique $\alpha$-invariant ideal $I$ of $\stackrel{\alpha}{A}$, where $\hat{\alpha}$ is the dual action of $\hat{G}$ on $A_{\alpha} \underset{\alpha}{ }$ ([T] $)$. Hence, there is an order isomorphism

$$
\Psi:\{I \triangleleft A \mid I \text { is } \alpha \text {-invariant }\} \rightarrow\{J \triangleleft A \underset{\alpha}{A \rtimes} \mid J \text { is } \hat{\alpha} \text {-invariant }\}
$$

given by

$$
\Psi(I):=\underset{\alpha}{\rtimes} G
$$

for each $\alpha$-invariant ideal $I$ of $A$.

Now, observe that by the above facts, it follows that the order isomorphisms $\Phi$ and $\Psi$, which have the same domains of definition, agree on their domain of definition and hence the range of $\Phi$ is equal to the range of $\Psi$. But this together with the above discussion imply that $\theta$ is an order isomorphism. The fact that $\{I \triangleleft A \mid I$ is $\alpha$-invariant $\}$ and $\{J \mid J \triangleleft A \underset{\alpha}{\rtimes} G\}$ are lattices and $\theta$ is a lattice isomorphism follows from [Pa4, Remark 4.3]. 
Now, suppose that $I \triangleleft A, I$ is $\alpha$-invariant and $I \subseteq\langle\mathcal{P}(\underset{\alpha}{\rtimes} G)\rangle$. This implies that $\theta(I)=I \rtimes G \subseteq\langle\mathcal{P}(I \rtimes G)\rangle=\langle\mathcal{P}(\theta(I))\rangle$, from which we conclude that $\theta(I)$ is generated by its projections. On the other hand, if $I \triangleleft A, I$ is $\alpha$-invariant and $\theta(I)=\underset{\alpha}{\underset{\alpha}{x} G}$ is generated by projections, then, clearly $I \subseteq \underset{\alpha}{{ }_{\alpha} G}=\langle\mathcal{P}(\underset{\alpha}{\rtimes} G)\rangle$. These facts, together with [Pa4, Remark 4.3], imply that $\theta$ induces canonically a lattice isomorphism

$$
\begin{aligned}
& \{I \triangleleft A \mid I \text { is } \alpha \text {-invariant and } I \subseteq\langle\mathcal{P}(\underset{\alpha}{\rtimes} G)\rangle\} \\
& \quad \cong\{J \triangleleft \underset{\alpha}{\rtimes} G \mid J \text { is generated by projections }\} .
\end{aligned}
$$

Corollary 2.2. Let $A, G$ and $\alpha$ be as in the hypothesis of Theorem 2.1. Then, the following are equivalent:

(a) $A \rtimes G$ has the ideal property.

(b) For every $\alpha$-invariant ideal $I$ of $A$, we have that $I \subseteq\langle\mathcal{P}(\underset{\alpha}{\rtimes} G)\rangle$.

Corollary 2.3. Let $A, G$ and $\alpha$ be as in the hypothesis of Theorem 2.1. Moreover, suppose that the spectrum of $A$ has dimension zero. Then, $A \rtimes G$ has the ideal property.

Proof. Since $A$ is a commutative, separable $C^{*}$-algebra with zero-dimensional spectrum, then $A$ is an $A F$ algebra. Hence, $A$ has the ideal property. Now, the result follows by also using Corollary 2.2 .

Corollary 2.4. Let $A, G$ and $\alpha$ be as in the hypothesis of Theorem 2.1. Suppose moreover that $A=C(X)$, where $X$ is a compact, Hausdorff topological space, and that every closed $\alpha$-invariant subset of $X$ is also open. Then, $A \rtimes G$ has the ideal property.

Proof. Let $I$ be an $\alpha$-invariant ideal of $A$. Then, there is a closed, $\alpha$-invariant subset $F$ of $X$ such that $I=\left\{f \in C(X) \mid f_{\mid F}=0\right\}$. Since $X \backslash F$ is closed (because, by hypothesis, $F$ is open) it follows that the map $A \ni f \longmapsto f_{\mid F} \oplus f_{\mid X \backslash F} \in$ $C(F) \oplus C(X \backslash F)$ is an isomorphism, which implies that $I \cong C(X \backslash F)$ and hence, $I$ being unital, is generated by projections. Now, the result follows by also using Corollary 2.2 .

Definition 2.5. Let $G$ be a locally compact group. We say that $G$ has the ideal property if its group $C^{*}$-algebra $C^{*}(G)$ has the ideal property.

Question (Shuzhou Wang). Let $(A, G, \alpha)$ be a $C^{*}$-dynamical system such that $A$ and $G$ have the ideal property. Does the crossed product $\underset{\alpha}{A \rtimes G}$ (always) have the ideal property?

The answer to the above question is "no", even in the "separable" case, as it follows from the following:

Theorem 2.6. There are $C^{*}$-dynamical systems $(A, G, \alpha)$ with $A$ a separable, simple, unital $C^{*}$-algebra (and hence, with the ideal property) and with $G$ a separable, commutative topological group with the ideal property such that $\underset{\alpha}{A \rtimes G}$ does not have the ideal property. 
The proof of the above theorem will use the following proposition:

Proposition 2.7. Let $G$ be a compact, abelian group. Then, $G$ has the ideal property.

Proof. The hypothesis implies that $\hat{G}$ (the dual group of $G$ ) is a discrete, abelian group. We have that $C^{*}(G) \cong C_{0}(\hat{G})$ (see $[\mathrm{Pe}$, Proposition 7.1.6]). On the other hand, since $\hat{G}$ is discrete, then $\operatorname{dim}(\hat{G})=0$ and hence $C_{0}(\hat{G})$ is an approximately finite-dimensional $C^{*}$-algebra, i.e., it is the inductive limit of a net of finite-dimensional $C^{*}$-algebras. Since a finite-dimensional $C^{*}$-algebra has the ideal property, this implies that $C_{0}(\hat{G})$ has the ideal property (as an inductive limit of $C^{*}$ algebras with the ideal property $([\overline{\mathrm{Pa} 4}$, Proposition 2.3]) $)$ and hence, by the above discussion, it follows that $C^{*}(G)$ has the ideal property, which ends the proof.

Proof of Theorem 2.6. Let $(B, \mathbb{Z}, \alpha)$ be a $C^{*}$-dynamical system with $B=C(X)$, where $X$ is an infinite, compact, connected, metrizable topological space and such that $A:=B \rtimes \mathbb{Z}$ is a simple $C^{*}$-algebra. (Clearly, $A$ is unital and separable and hence with the ideal property, since, obviously, every simple, unital $C^{*}$-algebra has the ideal property.) Note that $A$ is simple if (and only if) the homeomorphism of $X$ induced by $\alpha(1)$ is minimal (see e.g. [D Theorem VIII. 3.9]). (Remark that the irrational rotation algebras are examples of such kind of $C^{*}$-algebras $A([\overline{\mathrm{Bl}}])$.)

Now, by Takai duality $([\mathrm{T}])$, we have that

$$
\underset{\hat{\alpha}}{A \rtimes \mathbb{T}} \cong B \otimes \mathcal{K}
$$

where $\hat{\alpha}: \mathbb{T}=\hat{\mathbb{Z}} \rightarrow \operatorname{Aut}(A)$ is the dual action $([\mathbb{T}])$. Our claim is that $B \otimes \mathcal{K}$ does not have the ideal property and this fact together with the above isomorphism will imply that $A \rtimes \mathbb{T}$ does not have the ideal property either. Indeed, let $\phi \neq F=\bar{F} \varsubsetneqq X$ and let $I: \stackrel{\hat{\alpha}}{=}\left\{f \in B \otimes \mathcal{K}=C(X, \mathcal{K}) \mid f_{\mid F}=0\right\}$. Then, clearly $I \triangleleft B \otimes \mathcal{K}$ and $\mathcal{P}(I)=\{0\}$, since $X$ is connected, but $I$ is not generated by its projections (because, obviously, $I \neq\{0\})$. Hence, $B \otimes \mathcal{K}$ does not have the ideal property. The proof of the theorem is over if we observe that since $\mathbb{T}$ is a compact, abelian group, the separable group $G=\mathbb{T}$ has the ideal property by Proposition 2.7 .

\section{ACKNOWLEDGMENTS}

This material is based upon work supported by, or in part by, the U.S. Army Research Office under grant number DAAD19-00-1-0152. This research was also partially supported by NSF grant DMS-0101060.

\section{REFERENCES}

[Bl] B. Blackadar, K-theory for operator algebras, Second edition, Mathematical Sciences Research Institute Publications, 5, Cambridge University Press, Cambridge, 1998. MR 99g:46104

[Br] O. Bratteli, Inductive limits of finite dimensional $C^{*}$-algebras, Trans. Amer. Math. Soc. 171 (1972), 195-234. MR 47:844

[BPe] L. G. Brown and G. K. Pedersen, $C^{*}$-algebras of real rank zero, J. Funct. Anal. 99 (1991), no. 1, 131-149. MR 92m:46086

[D] K. Davidson, $C^{*}$-algebras by example, Fields Institute Monographs, 6, American Mathematical Society, Providence, RI, 1996. MR 97i:46095 
[EH] E. G. Effros and F. Hahn, Locally compact transformation groups and $C^{*}$-algebras, Memoirs of the American Mathematical Society, No. 75, American Mathematical Society, Providence, RI, 1967. MR 37:2895

[Ell] G. A. Elliott, The classification problem for amenable $C^{*}$-algebras, Proceedings of the International Congress of Mathematicians, Vol. 1, 2 (Zürich, 1994), 922-932, Birkhäuser, Basel, 1995. MR 97g:46072

[GL] E. C. Gootman and A. J. Lazar, Applications of non-commutative duality to crossed product $C^{*}$-algebras determined by an action or coaction, Proc. London Math. Soc. (3) 59 (1989), no. 3, 593-624. MR 91b:46069

[Pa1] C. Pasnicu, AH algebras with the ideal property, Operator algebras and operator theory (Shanghai, 1997), 277-288, Contemp. Math., 228, American Mathematical Society, Providence, RI, 1998. MR 99m:46141

[Pa2] C. Pasnicu, Extensions of AH algebras with the ideal property, Proc. Edinburgh Math. Soc. (2) 42 (1999), no. 1, 65-76. MR 2001c:46112

[Pa3] C. Pasnicu, Shape equivalence, nonstable $K$-theory and AH algebras, Pacific J. Math. 192 (2000), no. 1, 159-182. MR 2001d:46102

[Pa4] C. Pasnicu, On the AH algebras with the ideal property, J. Operator Theory 43 (2000), no. 2, 389-407. MR 2001c:46111

[Pa5] C. Pasnicu, On the (strong) GAH algebras, Rev. Roumaine Math. Pures Appl. 46 (2001), no. 4, 489-498.

[Pa6] C. Pasnicu, The ideal property and traces, Math. Nachr. 227 (2001), 127-132. MR 2002g: 46101

[Pa7] C. Pasnicu, Ideals generated by projections and inductive limit $C^{*}$-algebras, Rocky Mountain J. Math. 31 (2001), no. 3, 1083-1095.

[Pa8] C. Pasnicu, The projection property, Glasg. Math. J. 44 (2002), no. 2, 293-300.

[Pa9] C. Pasnicu, LB algebras, J. Operator Theory (to appear).

$[\mathrm{PaR}]$ C. Pasnicu and M. Rørdam, Tensor products of $C^{*}$-algebras with the ideal property, J. Funct. Anal. 177 (2000), no. 1, 130-137. MR 2001m:46124

[Pe] G. K. Pedersen, $C^{*}$-algebras and their automorphism groups, London Mathematical Society Monographs, 14, Academic Press, Inc. (Harcourt Brace Jovanovich, Publishers), LondonNew York, 1979. MR 81e:46037

[S] K. H. Stevens, The classification of certain non-simple approximate interval algebras, Fields Inst. Commun., 20, American Mathematical Society, Providence, RI, 1998. MR 2002d: 46052

[T] H. Takai, On a duality for crossed products of $C^{*}$-algebras, J. Funct. Anal. 19 (1975), 25-39. MR 51:1413

[Z] G. Zeller - Meier, Products croisés d'une $C^{*}$-algèbre par un groupe d'atuomorphismes, J. Math. Pures Appl. (9) 47 (1968), 101-239. MR 39:3329

Department of Mathematics and Computer Science, University of Puerto Rico, Box 23355, San Juan, Puerto Rico 00931-3355

E-mail address: cpasnic@upracd.upr.clu.edu 